\title{
Models and Algorithms for Diffuse Optical Tomographic System
}

\author{
Samir Kumar Biswas ${ }^{1}$, Rajan Kanhirodan ${ }^{1 *}$, Ram Mohan Vasu ${ }^{2}$ \\ ${ }^{1}$ Department of Physics, Indian Institute of Science, Bangalore, India \\ ${ }^{2}$ Department of Instrumentation and Applied Physics, Indian Institute of Science, Bangalore, India \\ Email: *rajan@physics.iisc.ernet.in, sameer2blore@gmail.com, vasu@isu.iisc.ernet.in
}

Received October 22, 2013; revised November 22, 2013; accepted November 29, 2013

Copyright (C) 2013 Samir Kumar Biswas et al. This is an open access article distributed under the Creative Commons Attribution License, which permits unrestricted use, distribution, and reproduction in any medium, provided the original work is properly cited.

\begin{abstract}
Diffuse optical tomography (DOT) using near-infrared (NIR) light is a promising tool for noninvasive imaging of deep tissue. The approach is capable of reconstructing the quantitative optical parameters (absorption coefficient and scattering coefficient) of a soft tissue. The motivation for reconstructing the optical property variation is that it and, in particular, the absorption coefficient variation, can be used to diagnose different metabolic and disease states of tissue. In DOT, like any other medical imaging modality, the aim is to produce a reconstruction with good spatial resolution and in contrast with noisy measurements. The parameter recovery known as inverse problem in highly scattering biological tissues is a nonlinear and ill-posed problem and is generally solved through iterative methods. The algorithm uses a forward model to arrive at a prediction flux density at the tissue boundary. The forward model uses light transport models such as stochastic Monte Carlo simulation or deterministic methods such as radioactive transfer equation (RTE) or a simplified version of RTE namely the diffusion equation (DE). The finite element method (FEM) is used for discretizing the diffusion equation. The frequently used algorithm for solving the inverse problem is Newton-based Model based Iterative Image Reconstruction (N-MoBIIR). Many Variants of Gauss-Newton approaches are proposed for DOT reconstruction. The focuses of such developments are 1) to reduce the computational complexity; 2) to improve spatial recovery; and 3) to improve contrast recovery. These algorithms are 1) Hessian based MoBIIR; 2) Broyden-based MoBIIR; 3) adjoint Broyden-based MoBIIR; and 4) pseudo-dynamic approaches.
\end{abstract}

Keywords: Diffuse Optical Tomography; Gauss Newton Methods; Broyden and Adjoint Broyden Approaches; Pseudo-Dynamic Method

\section{Introduction}

Diffuse Optical Tomography (DOT) provides an approach to probing highly scattering media such as tissue using low-energy near infra-red light (NIR) using the boundary measurements to reconstruct images of the optical parameter map of the media. Low power (1 - 10 milliwatt) NIR laser light, modulated by $100 \mathrm{MHz}$ sinusoidal signal is passed through a tissue, and the existing light intensity and phase are measured on the boundary. The predominant effects are the optical absorption and scattering. The transport of photons through a biological tissue is well established through diffusion equation [1-6] which models the propagation of light through the highly scattering turbid media.

The forward model frequently uses light transport

"Corresponding author. models such as stochastic Monte Carlo simulation [7] or deterministic methods such as radiative transfer equation (RTE) [8]. Under certain conditions such as $\left(\mu_{a} \ll \mu_{s}^{\prime}\right)$, the light transport problem can be simplified by the diffusion equation (DE) [9]. The RTE is the most appropriate model for light transport through an inhomogeneous material. The RTE has many advantages which include the possibility of modelling the light transport through an irregular tissue medium. However, it is computationally very expensive. So the DOT systems use the diffusion based approach. Gauss-Newton Method [2]is most frequently used for solving the DOT problem. The methods based on Monte-Carlo are perturbation reconstruction methods [10-12]. The numerical methods used for discretizing the DE are the finite difference method (FDM) [13], and the finite element method (FEM) [2]. Hybrid FEM models with RTE for locations close to the source 
and DE for others regions have also been considered [14]. The FEM discretization scheme considers that the solution region comprises many small interconnected tiny subregions and gives a piece wise approximation to the governing equation; i.e. the complex partial differential equation is reduced to a set of linear or non-linear simultaneous equations. Thus the reconstruction problem is a nonlinear optimization problem where the objective function defined as the norm of the difference between the model predicted flux and the actual measurement data for a given set of optical parameters is minimized. One method of overcoming the ill-posedness is to incorporate a regularization parameter. Regularization methods replace the original ill-posed problem with a better conditioned but related one in order to diminish the effects of noise in data and produce a regularized solution to the original problem.

A discretized version of diffusion equation is solved using finite element method (FEM) for providing the forward model for photon transport. The solution of the forward problem is used for computing the Jacobian and the simultaneous equation is solved using conjugate gradient search.

In this study, we look at many approaches used for solving the DOT problem. In DOT, the number of unknowns far exceeds the number of measurements. An accurate and reliable reconstruction procedure is essential to make DOT a practically relevant diagnostic tool. The iterative methods are often used for solving this type of both nonlinear and ill-posed problems based on nonlinear optimization in order to minimize a data-model misfit functional. The algorithm comprises a two-step procedure. The first step involves propagation of light to generate the so-called 'forward data' or prediction data and an update procedure that uses the difference between the prediction data and measurement data for the incremental parameter distribution. For the parameter update, one often uses a variation of Newton's method in the hope of producing the parameter update in the right direction leading to the minimization of the error functional. This involves the computation of the Jacobian of the forward light propagation equation in each iteration. The approach is termed as model based iterative image reconstruction (MoBIIR).

The DOT involves an intense computational block that iteratively recovers unknown discretized parameter vectors from partial and noisy boundary measurement data. Being ill-posed, the reconstruction problem often requires regularization to yield meaningful results. To keep the dimension of the unknown parameters vector within reasonable limits and thus ensure the inversion procedure less ill-posed and tractable, the DOT usually attempts to solve only 2-D problems, recovering 2-D cross-sections with which 3-D images could be built-up by stacking multiple 2-D planes. The most formidable difficulty in crossing over a full-blown $3 \mathrm{D}$ problem is the disproportionate increase in the parameter vector dimension (a typical tenfold increase) compared to the data dimension where one cannot expect an increase beyond 2 - 3 folds. This makes the reconstruction problem more severely ill-posed to the extent that the iterations are rendered intractably owing to larger null-spaces for the (discretized) system matrices. As the iteration progresses, the mismatch ( $\Delta M$, the difference between the computed and measurement value) decreases.

The main drawback of a Newton based MoBIIR algorithm (N-MoBIIR) is the computational complexity of the algorithm. The Jacobian computation in each iteration is the root cause of the high computation time. The Broyden approach is proposed to reduce the computation time by an order of magnitude. In the Broyden-based approach, Jacobian is calculated only once with uniform distribution of optical parameters to start with and then in each iteration. It is updated over the region of interest (ROI) only using a rank-1 update procedure.. The idea behind the Jacobian $(\mathrm{J})$ update is the step gradient of adjoint operator at ROI that localizes the inhomogeneities. The other difficulty with MoBIIR is that it requires regularization to ease the ill-posedness of the problem. However, the selection of a regularization parameter is arbitrary. An alternative route to the above iterative solution is through introducing an artificial dynamics in the system and treating the steady-state response of the artificially evolving dynamical system as a solution. This alternative also avoids an explicit inversion of the linearized operator as in the Gauss-Newton update equation and thus helps to get away with the regularization.

\section{Algorithms}

\subsection{Newton-Based Approach}

The light diffusion equation in frequency domain is,

$$
\begin{aligned}
& {\left[-\nabla \cdot k(r) \nabla+\left(\mu_{a}(r)+\frac{j \omega}{c}\right)\right] \phi(r, \omega)} \\
& =\left[A_{d c} \delta(\omega)+A_{a c} \delta\left(\omega-\omega_{0}\right)\right] \delta\left(r-r_{0}\right)
\end{aligned}
$$

where $\phi(r)$ is the photon flux, $k(r)$ is the diffusion coefficient and is given by

$$
k(r)=\left[3\left(\mu_{a}(r)+\mu_{s}^{\prime}(r)\right)\right]^{-1}
$$

$\mu_{a}$ and $\mu_{s}^{\prime}$ are absorption coefficient and reduced scattering coefficient $\left(\mu_{a} \ll \mu_{s}^{\prime}\right)$ respectively. The input photon is from a source of constant intensity $A_{d c}$ located at $r=r_{0}$. The transmitted output optical signal measured by a photomultiplier tube.

The DOT problem is represented by a non-linear operator given by $F(\mu)$ where $F$ gives model predicted 
data over the domain and $\boldsymbol{M}$ is the computed measurement vector obtained from $\left.\Phi\right|_{\Delta \Omega}$ and $\mu=\left(\mu_{a}, k\right)$.

$$
F(\mu)=M
$$

The image reconstruction problem seeks to find a solution $\left(\mu_{a}, k(r)\right)$ such that the difference between the model predicted $F\left(\mu_{a}, k\right)$ and the experimental measurement $\left(M^{E}\right)$ is minimum. To minimize the error, the cost functional $\chi\left(\mu_{a}, \kappa\right)$ is minimized and the cost functional is defined as [1];

$$
\chi\left(\mu_{a}, k\right)=\arg \left\{\min _{\mu_{a}, k}\left\|\left[M^{E}-F\left(\mu_{a}, k\right)\right]\right\|\right\}
$$

where $\|$.$\| is L_{2}$ norm. Through Gauss-Newton and Levenberg-Marquardt $[1,15,16]$ algorithms, the minimized form of the above equation is given as,

$$
\left(\Delta \mu_{a}, \Delta k\right)=\left[J^{T} J+\lambda I\right]^{-1} J^{T} \Delta M
$$

where $\Delta M$ is the difference between model predicted data $\left[M^{C}=F(\mu)\right]$ and experimental measurement data $\left(M^{E}\right)^{2}$, and $\mathrm{J}$ is the Jacobian matrix which has been evaluated at each iteration of MoBIIR algorithm (Figure 1). The above equation is the parameter update expression. In Equation 5, I is the identity matrix whose dimension is equal to the dimension of $\mathrm{J}^{T} \mathrm{~J} . \lambda$ is regularization parameter and the order of magnitude of $\lambda \mathrm{I}$ should be near to that of $\mathrm{J}^{T} \mathrm{~J}$. The impact of noise and $\lambda$ on the reconstruction is discussed in results section. The Figure 1 gives a flow chart of the approach based on Gauss Newton.

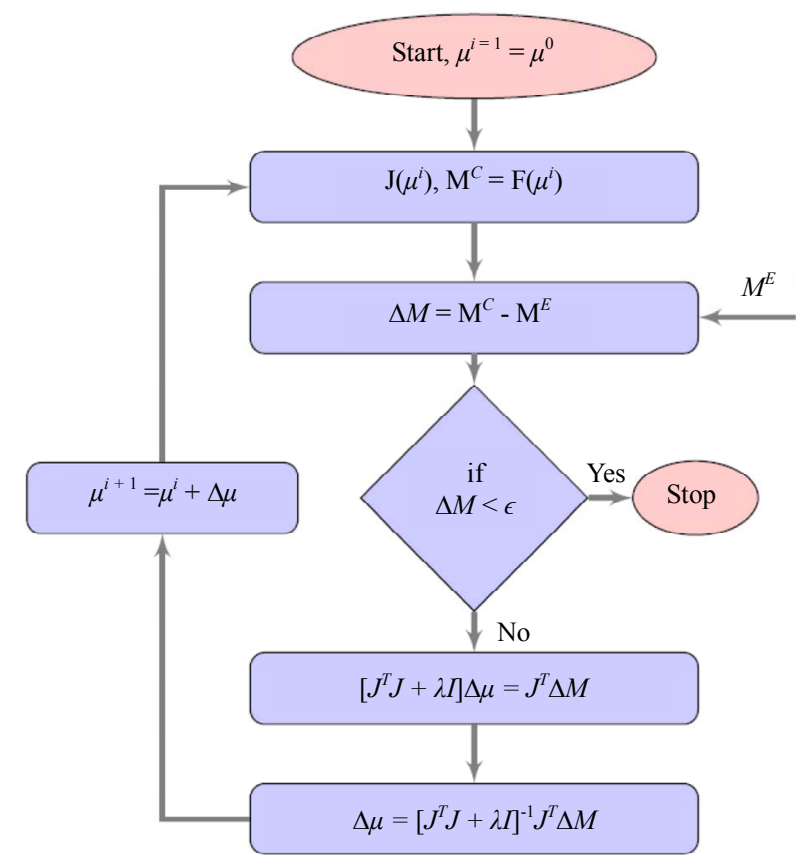

Figure 1. Flowchart for image reconstruction by Newton's method based MoBIIR algorithm.

\subsection{Hessian Based Approach}

The iterative reconstruction algorithm recovers an approximation to $\mu$ from the set of boundary measurements $M^{e}$. By directly Taylor expanding Equation 3, and using a quadratic term involving Hessian, the perturbation equation can be written as,

$$
\begin{gathered}
f\left(\mu_{i+1}\right)=f\left(\mu_{i}\right)+\Delta \mu_{i} \cdot F^{\prime}\left(\mu_{i}\right) \\
+\Delta \mu_{i}^{T} \cdot F^{\prime \prime}\left(\mu_{i}\right) \Delta \mu_{i}
\end{gathered}
$$

where $F^{\prime \prime}$ is the Hessian corresponding to the measurement. For $d$ number of detectors, the above equation can be rewritten as,

$$
\left[F^{\prime T} F^{\prime} \Delta \mu-\sum_{i=1}^{d} F_{i}^{\prime \prime} \Delta M_{i} \Delta \mu\right]=F^{\prime T} \Delta M .
$$

The Equation 7 is the update equation obtained from the quadratic perturbation equation. The term $F^{\prime \prime} \Delta M$ is often observed to be diagonally dominant and can be denoted by $\lambda_{i i}$, neglecting the off diagonal terms. Thus, through the incorporation of the second derivative term, the update equation has a generalized form with a physically consistent regularization term.

\subsection{Broyden Approaches}

The major constraint of Newton's method is the computationally expensive Jacobian evaluation $[17,18]$. The quasi-Newton methods provide an approximate Jacobian [19]. Samir et al [5] has developed an algorithm making use of Broyden's method [17,18,20] to improve the Jacobian update operation, which happens to be the major computational bottleneck of Newton-based MoBIIR. Broyden's method approximates the Newton direction by using an approximation of the Jacobian which is updated as iteration progresses. Broyden method uses the current estimate of the Jacobian $J_{i-1}$ and improves it by taking the solution of the secant equation that is a minimal modification to $J_{i-1}$. For this purpose one may apply rank-one updates. We have assumed that we have a nonsingular matrix $J\left(\mu^{i}\right)$ and we wish to produce an approximate $J\left(\mu^{i+1}\right)$ through rank-1 updates [21]. The forward solution can be expressed in terms of derivatives of the forward solution using Taylor expansion as,

$$
F\left(\mu^{i}\right)=F\left(\mu^{i+1}\right)+J\left(\mu^{i+1}\right)\left[\mu^{i}-\mu^{i+1}\right] .
$$

The Broyden's Jacobian update equation becomes

$$
\begin{aligned}
& J_{i+1}=J_{i}+\frac{\left[\Delta M^{i}-J_{i} \Delta \mu^{i}\right] \Delta \mu^{i T}}{\left[\Delta \mu^{i} \cdot \Delta \mu^{i}\right]} \\
& J_{i+1}=J_{i}+\Delta J_{i} \quad \text { where } \quad \Delta J_{i}=\frac{\left[\Delta M^{i}-J_{i} \Delta \mu^{i}\right] \Delta \mu^{i T}}{\left[\Delta \mu^{i} \cdot \Delta \mu^{i}\right]} .
\end{aligned}
$$


Equation 9 is referred to as Broyden's update equation. In Broyden's method there is no clue about the initial Jacobian estimate [22]. The initial value of Jacobian $\left[J\left(\mu^{0}\right)\right]$ is computed through analytical methods based on adjoint principles. It is found that since Jacobian update is only approximate, the number of iterations required by Broyden method for convergence is higher than that of gauss-Newton methods. This can be improved by re-calculating Jacobian using adjoint method when Jacobian is found to be outside the confidence domain (when MSE of the current estimate is less than MSE of the previous estimate). If the initial guess $\mu^{0}$ is sufficiently close to the actual optical parameter $\mu^{*}$ then the $J\left(\mu^{0}\right)$ is sufficiently close to $A\left(\mu^{0}\right)$ and the solution converges q-superlinearly to $\mu^{*}$. The most notable feature of Broyden approach is that it avoids direct computation of Jacobian, thereby providing a faster algorithm for DOT reconstruction.

\subsection{Adjoint Broyden Based MoBIIR}

Least change secant based Adjoint Broyden [23] update method is another approach for updating the system Jacobian approximately.

The direct and adjoint tangent conditions are

$$
J_{i+1} \Delta \mu^{i}=F^{\prime}\left(\mu^{i+1}\right) \Delta \mu^{i} \text { and } \sigma_{i}^{T} J_{i+1}=\sigma_{i}^{T} F^{\prime}\left(\mu^{i+1}\right)
$$

respectively. With respect to the Frobenius norm $\|\cdot\|_{F r o b}$, the least change update of a matrix $J_{i}$ to a matrix $J_{i+1}$ satisfies the direct secant condition $J_{i+1} \Delta \mu^{i}=\Delta M^{i}$ and the adjoint secant condition $\sigma_{i}^{T} J_{i+1}=\xi_{i}^{T}$, for normalized directions $\Delta \mu^{i}$ and $\sigma_{i}$, and is given as [23]

$$
J_{i+1}=J_{i}+r^{i} \Delta \mu^{i T}+\sigma_{i} \rho^{i T}-\sigma_{i} \sigma_{i}^{T} r^{i} \Delta \mu^{i T}
$$

where $r^{i}=\Delta M^{i}-J_{i} \Delta \mu^{i}, \quad \rho^{i}=\xi_{i}-J_{i}^{T} \sigma_{i}$. The rank-1 update for Jacobian update based on adjoint method is given as [5],

$$
J_{i+1}=J_{i}+\frac{\sigma_{i} \sigma_{i}^{T}}{\sigma_{i}^{T} \sigma_{i}}\left[F\left(\mu^{i+1}\right)^{\prime}-J_{i}\right] .
$$

The Adjoint Broyden update is categorized based on the choice of $\sigma_{i}$. For simplicity, we consider only secant direction [23] which is defined as,

$$
\begin{aligned}
& \sigma_{i}=\left[F\left(\mu^{i+1}\right)-F\left(\mu^{i}\right)\right] / \alpha_{i}-J_{i} \Delta \mu^{i} \\
& \text { with } \mu^{i+1}=\mu^{i}+\alpha_{i} \Delta \mu^{i} .
\end{aligned}
$$

where $\alpha_{i}$ is the step size and is estimated through line search method. The above equation has been solved in Adjoint Broyden based MoBIIR image reconstruction.

The image reconstruction flowchart using Broyden based MoBIIR is shown in Figure 2. The Jacobian is updated through Equation 9 and Equation 11 for Broyden method and adjoint Broyden method respectively.

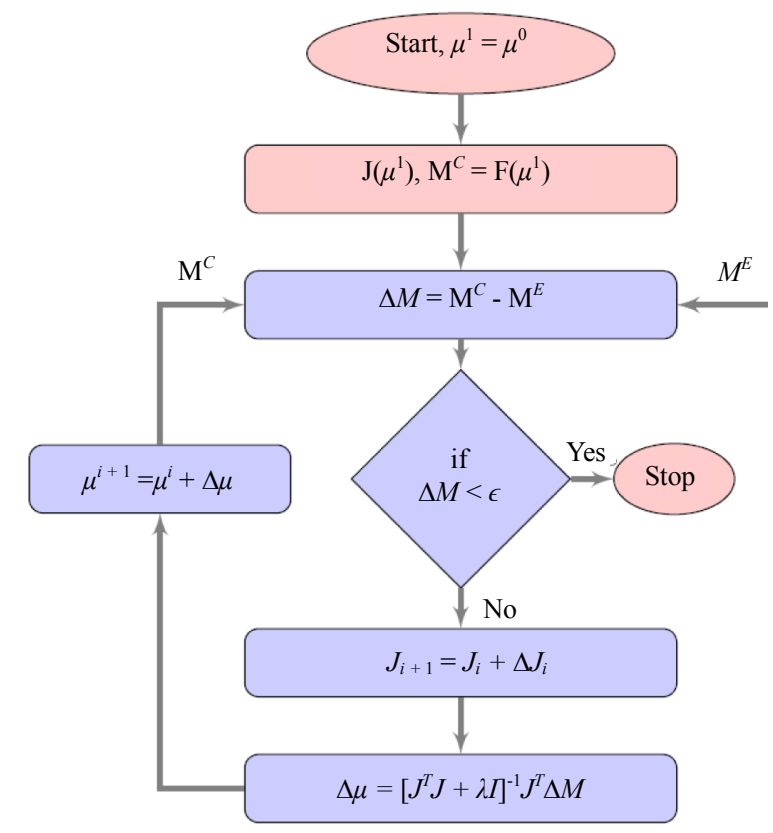

Figure 2. Flowchart for image reconstruction by Broydenbased MoBIIR (Equation 9) algorithm.

\subsection{Pseudo-Dynamic Approaches}

Diffuse optical tomographic imaging is an ill-posed problem, and a regularization term is used in image reconstruction to overcome this limitation. Several regularization schemes have been proposed in the literature [24]. However, choosing the right regularization parameter is a tedious task. A some what regularizationinsensitive route to computing the parameter updates using the normal equations Equation 5 or Equation 7 is to introduce an artificial time variable $[25,26]$. Such pseudodynamical systems, typically in the form of ordinary differential equations (ODE-s), may then be integrated and the updated parameter vector recovered once either a pseudo steady-state is reached or a suitable stopping rule is applied to the evolving parameter profile (the latter being necessary if the measured data are few and noisy). Samir et al [5] have used the above approach to arrive at a DOT image reconstruction.

For the DOT problem, the pseudo-time linearized ODE-s for the Gauss-Newton's normal equation for $t \in\left[t_{i}, t_{i}+\Delta t\right]$ is given by:

$$
\dot{\mu}+S\left(\mu^{i}, \lambda\right)\left[\mu(t)-\mu^{i}\right]+V_{s}=0
$$

where $\dot{\mu}:=\frac{\mathrm{d} \mu}{\mathrm{d} t}, \mu^{i}:=\mu\left(t_{i}\right)$,

$$
V_{s}\left(\mu^{i}\right):=F^{\prime T}\left(\mu^{i}\right)\left[M^{E}-F\left(\mu^{i}\right)\right] \text {, }
$$

and

$$
S\left(\mu^{i}, \lambda\right)=F^{\prime}\left(\mu^{i}\right) F^{\prime}\left(\mu^{i}\right)+\lambda I,
$$


when we use Equation 5. For the case where the quadratic perturbation is used (Equation 7), then $\mathrm{S}$ is replaced by

$$
G\left(\mu^{i}, \lambda\right)=F^{\prime T} F^{\prime}+F^{\prime T} \Delta \mu^{T} F^{\prime \prime}+\lambda I
$$

We first consider the linear case wherein Equation 5 is used to arrive at the pseudo-dynamic system. While initiating the pseudo-time recursion for $t \in\left[t_{i}, t_{i}+\Delta t\right]$, the initial parameter vector $\mu^{0}$ may be taken corresponding to the background value which is assumed to be known. Equation 13 may be integrated in closed-form leading to the following pseudo-time evolution,

$$
\begin{aligned}
\mu^{i+1} & =\exp \left(-S\left(\mu^{i}, \lambda\right) h_{i}\right) \mu^{i} \\
& +\int_{t_{i}}^{t_{i+1}} \exp \left(-S\left(\mu^{i}, \lambda\right)\left(t_{i+1}-t\right)\right) f(t) \mathrm{d} t
\end{aligned}
$$

where $f(t)=S\left(\mu^{i}, \lambda\right) \mu^{i}-V_{s}\left(\mu^{i}\right)$ and $h_{i}=t_{i+1}-t_{i}$. In the ideal case, when the measured data is noise-free, the sequence $\mu^{i} ; i=1,2, \cdots, N$ has a limit point $\hat{\mu}$, which yields the desired reconstruction. In a practical scenario where the measured data is noisy, i.e, $M^{E}=M^{E}(\delta)$ with $\delta$ being a measure of the noise 'strength'. In this case, a stopping rule may have to be imposed so that the sequence $\left\{\mu^{i} ; i=1,2, \cdots, N\right\}$ is stopped at $t^{*}=t_{N}\left(t^{*}\right.$

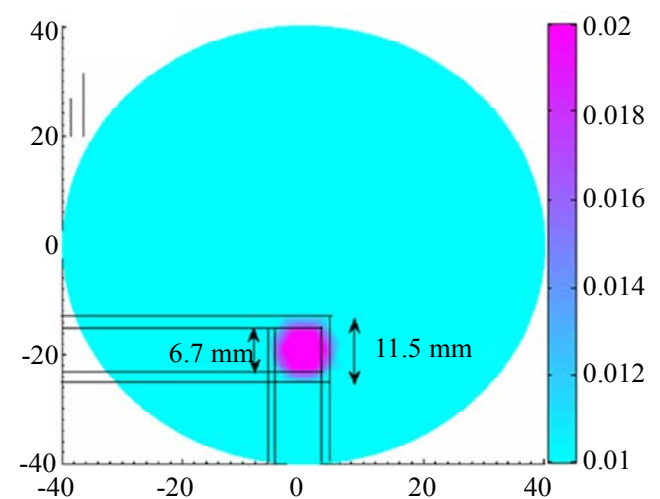

(a)

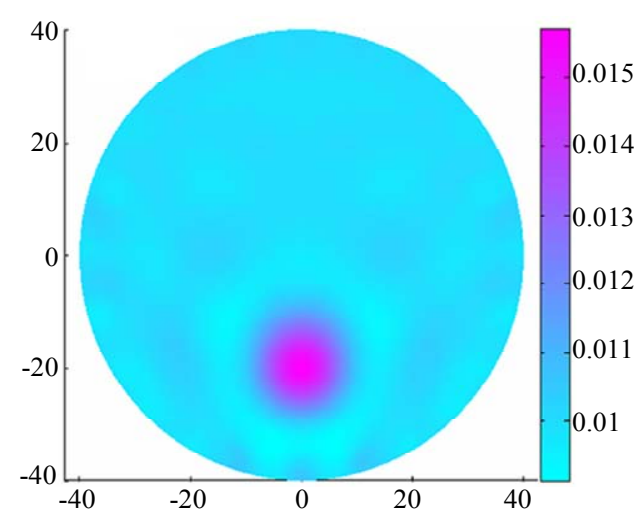

(c) is the stopping time) with $\hat{\mu} \cong \mu^{N}$.

\section{Results}

Figure 3 gives the reconstruction results with a single embedded inhomogeneity. Figure 3(a) is the phantom with one inhomogeneity. The reconstructed images using Newton-based MoBIIR, Broyden-based MoBIIR and adjoint Broyden-based MoBIIR are given in (b), (c), and (d) respectively.

Figure 4 gives the performance of the algorithm. It is seen that adjoint Broyden converges faster compared to other algorithms. Figure 4(a) shows that Newton, Broyden and adjoint Broyden methods converge at $16^{\text {th }}$, $22^{\text {th }}$ and $20^{\text {th }}$ iterations respectively. The cross section through the center of the inhomogeneities is shown in Figure 4(b).

Figure 5 gives the reconstruction results with two embedded inhomogeneities. Figure 5(a) is the phantom. The reconstructed images using Newton-based MoBIIR, Broyden-based MoBIIR and adjoint Broyden-based MoBIIR are given in (b), (c), and (d).

Figure 6 gives the performance of the algorithm with two inhomogeneities. MSE of reconstructed images with two inhomogeneities is shown in Figure 6(a). Figure 6(b)
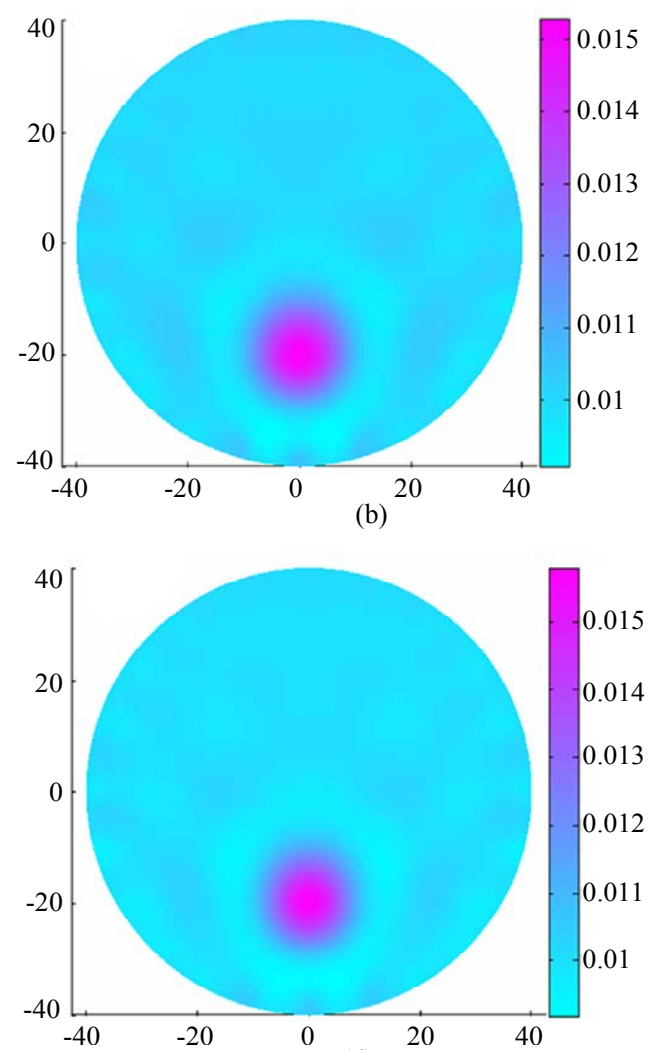

(d)

Figure 3. (a) Phantom with one inhomogeneity having $\mu_{a}=0.01 \mathrm{~mm}^{-1}$ and $\mu_{s}=1 \mathrm{~mm}^{-1}$ of size $8.2 \mathrm{~mm}$ at $(0,19.2)$. Reconstructed images using; (b) Newton-based MoBIIR; (c) Broyden-based MoBIIR; (d) Adjoint Broyden-based MoBIIR. 


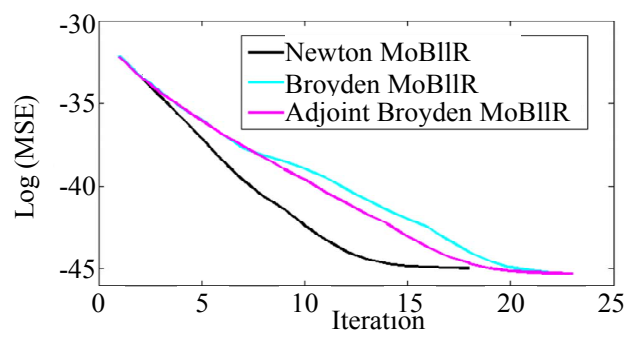

(a)

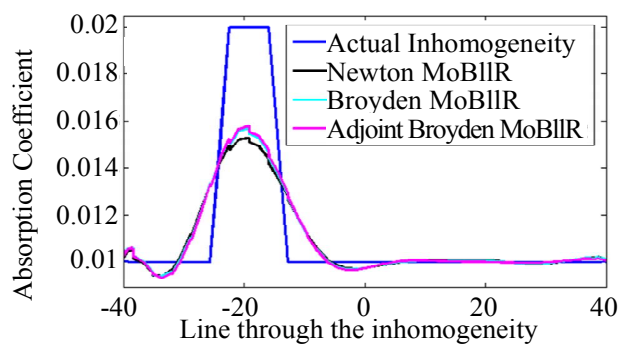

(b)

Figure 4. (a) Newton, Broyden and adjoint Broyden methods. They converge at $16^{\text {th }}, 22^{\text {th }}$ and $20^{\text {th }}$ iterations respectively; (b) Cross-section through the center of inhomogeneities for Newton, Broyden and adjoint Broyden methods.

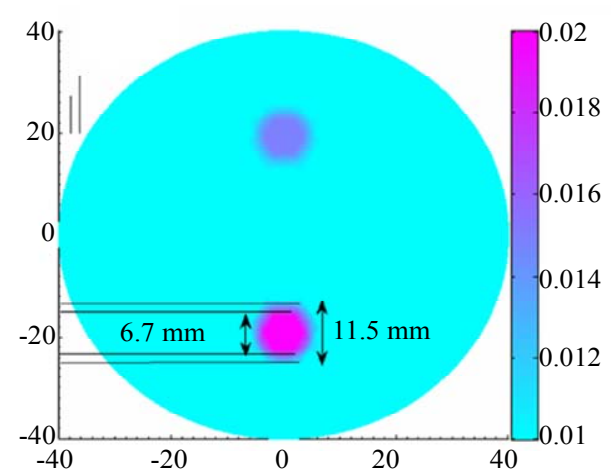

(a)

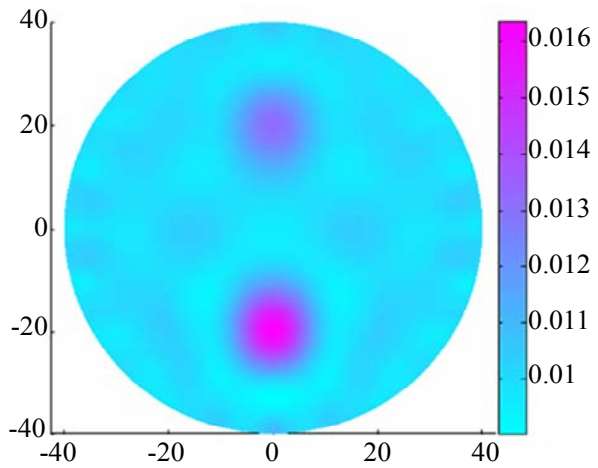

(c)

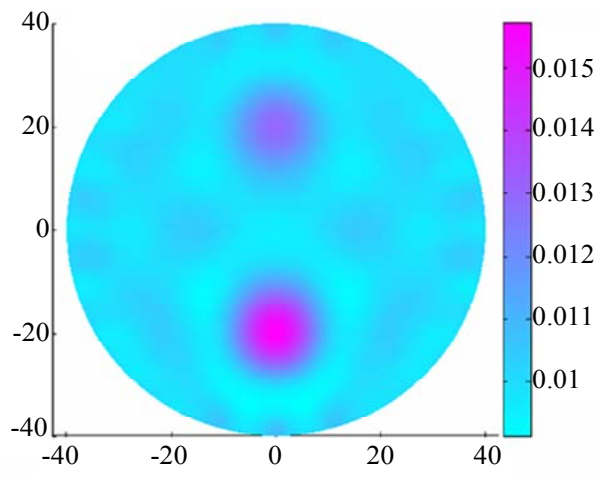

(b)

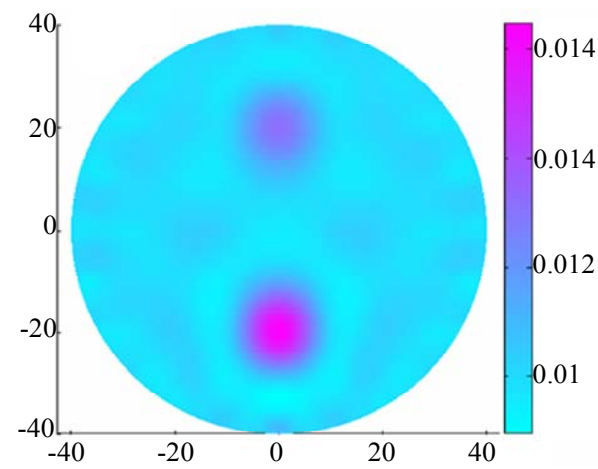

(d)

Figure 5. (a) Original simulated phantom with two inhomogeneities; The $\mu_{a}$ of the inhomogeneities are 0.02 and 0.015 $\mathrm{mm}^{-1}$ and are at $(0,-192.2)$ and $(0,19.2)$ respectively Reconstructed results using; (b) Newton; (c) Broyden; (d) adjoint Broyden method.

shows that Newton, Broyden and adjoint Broyden methods converge at $18^{\text {th }}, 37^{\text {th }}$ and $24^{\text {th }}$ iterations respectively. The line plot through the center of the inhomogeneities is shown in Figure 6(c).

Figure 7 gives the reconstruction results with two embedded inhomogeneities. Figure 7(a) is the reconstructed image by Gauss-Newton method. Figures 7(b) and (c) are the reconstructed images by linear pseudo-dynamic time marching algorithm and by non-linear (Hessian integrated) pseudo-dynamic time marching algorithm respectively.

Figure 8 analyzes the results. The line plot through the center of inhomogeneities is shown in Figure 8(a). The variation of the Normalized Mean Square Error (MSE) with Iteration is shown in Figure 8(b). The blue line represents Gauss-Newton's method and green line represents pseudo dynamic time matching algorithm.

\section{Conclusion}

In this study, we look at many approaches used for solving the DOT problem. Like any medical image reconstruction algorithms, the main focus is to reconstruct a high resolution image with good contrast. Since the 


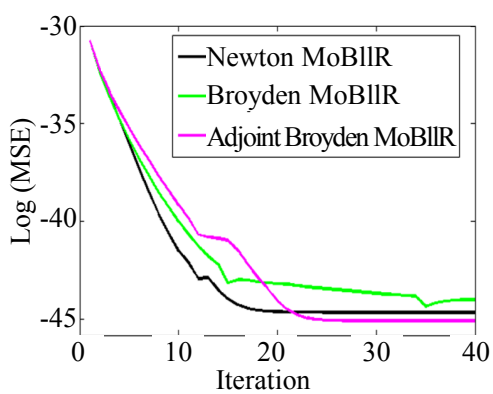

(a)

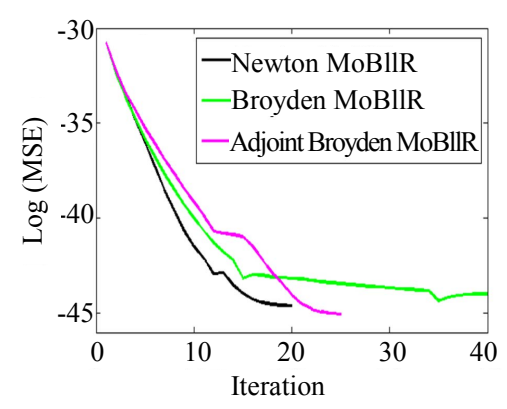

(b)

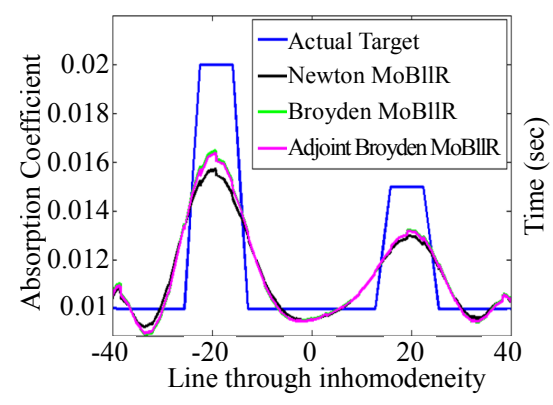

(c)

Figure 6. (a) MSE of reconstructed images with two inhomogeneities; (b) Newton, Broyden and adjoint Broyden methods converge at $18^{\text {th }}, 37^{\text {th }}$ and $24^{\text {th }}$ iterations respectively; (c) Line plot through the center of the inhomogeneities using Newton's and proposed algorithms.

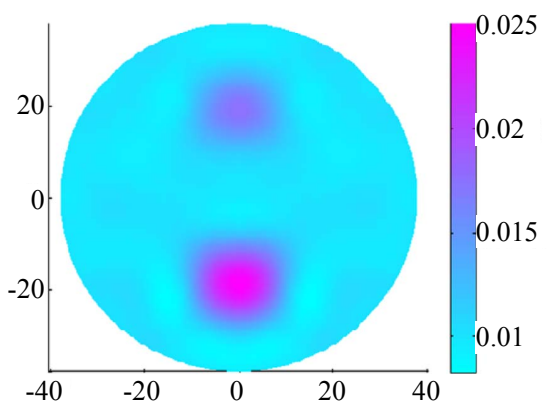

(a)

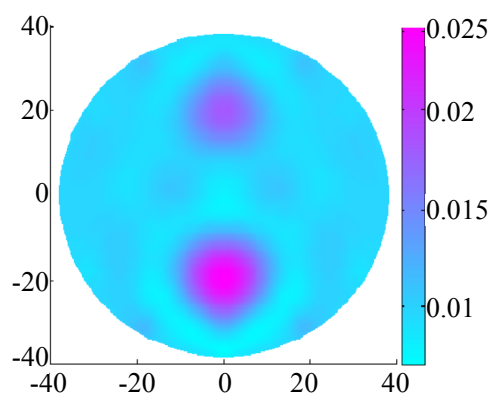

(b)

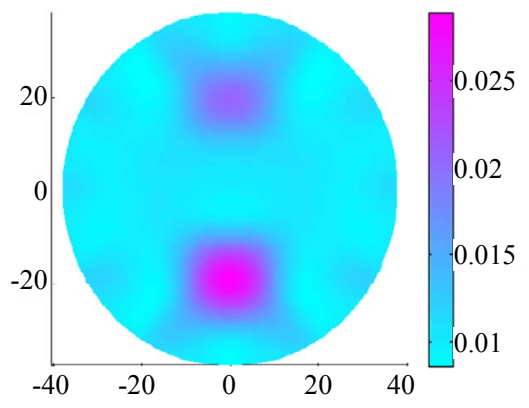

(c)

Figure 7. (a) Reconstructed image by Gauss-Newton method; (b) Reconstructed image by Linear pseudo-dynamic time marching algorithm; (c) Reconstructed image by non-linear (Hessian integrated) pseudo-dynamic time marching algorithm

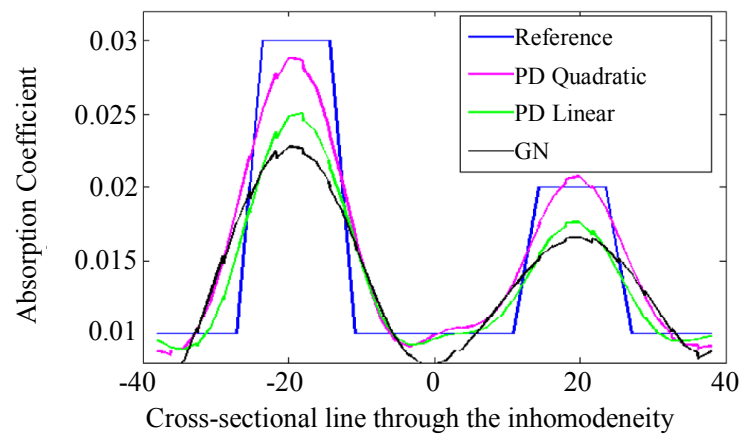

(a)

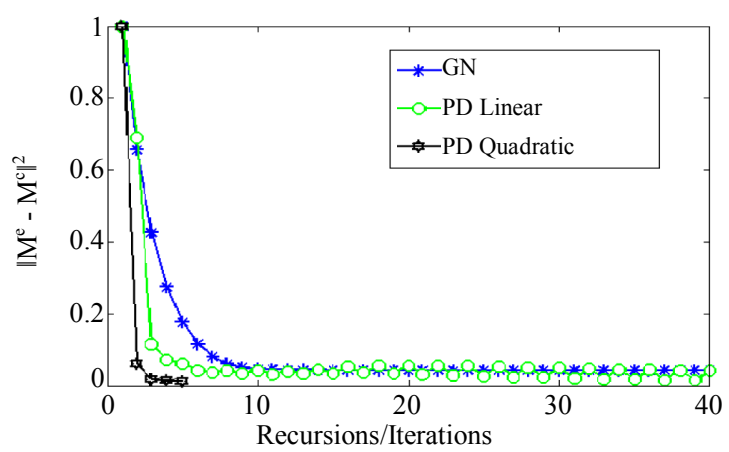

(b)

Figure 8. (a) The cross-sectional line plot through reconstructed inhomogeneity; (b) The variation of the Normalized Mean Square Error (MSE) with Iteration. The blue line represents Gauss-Newton's method and green line represents pseudo dynamic time matching algorithm.

problem is non-linear and ill-posed, the iterative methods are often used for solving this type of problems. We have summarized a few studies we undertook towards this. They are 1) Gauss-Newton based MoBIIR; 2) Quadratic Gauss-Newton, Broyden-based MoBIIR; 3) Adjoint Broyden based MoBIIR, and pseudo-dynamic approaches.

\section{REFERENCES}

[1] S. R. Arridge, "Optical Tomography in Medical Imag- ing," Inverse Problems, Vol. 15, No. 2, 1999, pp. R41R93.

[2] S. R. Arridge, M. Schweiger, M. Hiraoka and D. T. Delpy, "Finite Element Approach for Modelling Photon Transport in Tissue," Medical Physics, Vol. 20, 1993, pp. 299309. http://dx.doi.org/10.1118/1.597069

[3] B. Kanmani and R. M. Vasu, "Noise-Tolerance Analysis for Detection and Reconstruction of Absorbing Inhomogeneities with Diffuse Optical Tomography Using Single- and Phase-Correlated Dual-Source Schemes," Physics in Medicine and Biology, Vol. 52, 2007, p. 1409. 
http://dx.doi.org/10.1088/0031-9155/52/5/013

[4] B. W. Pogue, S. C. Davis, X. Song, B. A. Brooksby, H. Dehghani and K. D. Paulsen, "Image Analysis Methods for Diffuse Optical Tomography," Journal of Biomedical Optics, Vol. 11, 2006, Article ID: 1033001. http://dx.doi.org/10.1117/1.2209908

[5] S. K. Biswas, K. Rajan, R. M. Vasu and D. Roy, "Accelerated Gradient Based Diffuse Optical Tomographic Image Reconstruction," Medical Physics, Vol. 38, 2011, p. 539. http://dx.doi.org/10.1118/1.3531572

[6] S. K. Biswas, K. Rajan and R. M. Vasu, "Practical Fully 3-D Reconstruction Algorithm for Diffuse Optical Tomography," Journal of the Optical Society of America A, Vol. 29, 2012, p. 1017. http://dx.doi.org/10.1364/JOSAA.29.001017

[7] D. A. Boas, J. P. Culver, J. J. Stott and A. K. Dunn, "Three Dimensional Monte Carlo Code for Photon Migration through Complex Heterogeneous Media Including the Adult Human Head," Optics Express, Vol. 10, No. 3, 2002, pp. 159-170. http://dx.doi.org/10.1364/OE.10.000159

[8] G. S. Abdoulaev and A. H. Hielscher, "Three-Dimensional Optical Tomography with the Equation of Radiative Transfer," Journal of Electronic Imaging, Vol. 12, No. 4, 2003, pp. 594-601. http://dx.doi.org/10.1117/1.1587730

[9] M. Schweiger, S. R. Arridge and I. Nissila, "GaussNewton Method for Image Reconstruction in Diffuse Optical Tomography," Physics in Medicine and Biology, Vol. 50, No. 10, 2005, pp. 2365-2386. http://dx.doi.org/10.1088/0031-9155/50/10/013

[10] C. K. Hayakawa and J. Spanier, F. Bevilacqua, A. K. Dunn, J. S. You, B. J. Tromberg and V. Venugopalan "Perturbation Monte Carlo Methods to Solve Inverse Photon Migration Problems in Heterogeneous Tissues," Optics Letters, Vol. 26, No. 17, 2001, pp. 1335-1337.

[11] P. K. Yalavarthy, K. Karlekar, H. S. Patel, R. M. Vasu, M. Pramanik, P. C. Mathias, B. Jain and P. K. Gupta, "Experimental Investigation of Perturbation Monte-Carlo Based Derivative Estimation for Imaging Low-Scattering Tissue," Optics Express, Vol. 13, No. 3, 2005, pp. 985988.

[12] A. Sassaroli, "Fast Perturbation Monte Carlo Method for Photon Migration in Heterogeneous Turbid Media," Optics Letters, Vol. 36, No. 11, 2011, pp. 2095-2097. http://dx.doi.org/10.1364/OL.36.002095

[13] B. W. Pogue, M. S. Patterson, H. Jiang and K. D. Paulsen, "Initial Assessment of a Simple System for Frequency Domain Diffuse Optical Tomography," Physics in Medicine and Biology, Vol. 40, 1995, pp. 1709-1729. http://dx.doi.org/10.1088/0031-9155/40/10/011

[14] T. Tarvainen, M. Vauhkonen, V. Kolemainen, S. R. Arridge and J. P. Kaipio, "Coupled Radiative Transfer
Equation and Diffusion Approximation Model for Photon Migration in Turbid Medium with Low-Scattering and Non-Scattering Regions," Physics in Medicine and Biology, Vol. 50, 2005, pp. 4913-4930.

http://dx.doi.org/10.1088/0031-9155/50/20/011

[15] K. Levenburg, "A Method for the Solution of Certain Non-Linear Problems in Least-Squares," Quarterly of Applied Mathematics, Vol. 2, 1944, p. 164.

[16] D. W. Marquardt, "An Algorithm for the Least-Square Estimation of Non-Linear Parameters," SIAM Journal on Applied Mathematics, Vol. 11, 1963, p. 431. http://dx.doi.org/10.1137/0111030

[17] C. G. Broyden, "On the Discovery of the Good Broyden Method," Mathematical Programming, Vol. 87, No. 2, 2000, p. 209.

[18] C. G. Broyden, "A Class of Methods for Solving Nonlinear Simultaneous Equations," Mathematics of Computation, Vol. 19, 1965, pp. 577-593.

[19] H. Wang and R. P. Tewakson, "Quasi-Gauss-Newton Method for Solving Non-Linear Algebraic Equations," Computers \& Mathematics with Applications, Vol. 25, 1993, pp. 53-63.

[20] R. H. Byrd, H. Khalfan and R. B. Schnabel, "A Theoretical and Experimental Study of the Symmetric Rank One Update," Technical Report CU-CS-489-90, University of Colorado, 2002

[21] J. Branes, "An Algorithm for Solving Nonlinear Equations Based on the Secant Method," Computer Journal, Vol. 8, No. 1, 1965, pp. 66-72.

[22] J. E. Dennis Jr. and R. B. Schnabel, "Numerical Methods for Unconstrained Optimization and Nonlinear Equations," Prentice-Hall, Englewood Cliffs, 1983.

[23] S. Schlenkrich, A. Griewank and A. Walther, "On the Local Convergence of Adjoint Broyden Methods," $\mathrm{Ma}$ thematical Programming, Vol. 121, No. 2, 2010, pp. 221247.

[24] B. W. Pogue, T. McBride, J. Prewitt, U. L. Osterberg and K. D. Paulsen, "Spatially variant regularization improves diffuse optical tomography," Applied Optics, Vol. 38, 1999 , pp. 2950-2961 http://dx.doi.org/10.1364/AO.38.002950

[25] B. Banerjee and D. Roy and R. M. Vasu, "A PseudoDynamical Systems Approach to a Class of Inverse Problems in Engineering," Proceedings of the Royal Society A, Vol. A465, 2009, pp. 1561-1579.

[26] B. Banerjee and D. Roy and R. M. Vasu, "A PseudoDynamic Sub-Optimal Filter for Elastography under Static Loading and Measurement," Physics in Medicine and Biology, Vol. 54, 2009, pp. 285-305. http://dx.doi.org/10.1088/0031-9155/54/2/008 